\title{
Deciphering the tête-à-tête between the microbiota and the immune system
}

\author{
Neeraj K. Surana ${ }^{1,2}$ and Dennis L. Kasper ${ }^{2}$ \\ 'Division of Infectious Diseases, Department of Medicine, Boston Children's Hospital, Boston, Massachusetts, USA. ²Department of Microbiology and Immunobiology, Harvard Medical School,
} Boston, Massachusetts, USA.

\begin{abstract}
The past decade has witnessed an explosion in studies - both clinical and basic science - examining the relationship between the microbiota and human health, and it is now clear that the effects of commensal organisms are much broader than previously believed. Among the microbiota's major contributions to host physiology is regulation of the development and maintenance of the immune system. There are now a handful of examples of intestinal commensal bacteria with defined immunomodulatory properties, but our mechanistic understanding of how microbes influence the immune system is still in its infancy. Nevertheless, several themes have emerged that provide a framework for appreciating microbe-induced immunoregulation. In this Review, we discuss the current state of knowledge regarding the role of the intestinal microbiota in immunologic development, highlighting mechanistic principles that can guide future work.
\end{abstract}

In his timeless 1926 classic Microbe Hunters, Paul de Kruif colorfully describes the herculean efforts of some of the greatest 19thcentury microbiologists and immunologists - Robert Koch, Louis Pasteur, and Élie Metchnikoff, among others - in establishing the concept that microorganisms and the host immune system live in a constant struggle against each other (1). These pioneers from medicine's "heroic age" (circa 1860-1910) laid the groundwork for what became the leitmotif of 20th-century microbiology research: a molecular dissection of microbial pathogenesis and its intersections with the immune system. However, even at the beginning of the 20th century, there were some who argued that the notoriety enjoyed by "pathogenic" bacteria was diverting attention from much-needed studies on commensal organisms (2).

\section{Relevance of the microbiota to human health}

In part because of the confluence of emerging and re-emerging technologies (e.g., high-throughput sequencing and the use of gnotobiotic animal models), tremendous advances have been made over the past decade toward a better understanding of how human health is influenced by the microbiota - the trillions of bacteria, viruses, fungi, and Archaea that colonize humans and, in fact, outnumber human cells in the body 10- to 100-fold. The prevailing view that has arisen from these studies is that, rather than waging a continual battle with each other, the host and its microbiota exist in a carefully negotiated state of détente in which each side requires the other. The host provides an ecologic niche and nutrient source for the microbiota, and, in turn, the commensal organisms contribute to host physiology by aiding in proper development of the intestine, processing of nutrients, protection from exogenous pathogens, and maturation of the immune system (3-8). If, however, this delicately balanced system is per-

Conflict of interest: The authors have declared that no conflict of interest exists. Reference information: J Clin Invest. 2014;124(10):4197-4203. doi:10.1172/JCI72332. turbed, a state termed "dysbiosis" may result: the composition of the microbiota may become dysregulated, potentially predisposing the host to a number of diseases marked by aberrant immune responses (e.g., inflammatory bowel disease, multiple sclerosis, asthma, type 1 diabetes, cancers) (9-16). In the hope that these dysbiotic microbial communities will provide insight into disease pathogenesis and uncover novel treatment modalities, numerous studies have examined differences in the microbiota of patients with or without a given disease process. Remarkably, virtually all of these studies have demonstrated differences in the microbiota between patient groups.

Nevertheless, because these investigations are typically designed as case-control studies rather than prospective investigations, it remains unclear whether the documented bacterial associations are a direct cause - or a consequence - of the underlying disease process. Although the case-control approach is typically correlative in nature, it has been used to identify candidate organisms that may affect disease susceptibility and immune responses. In one study, for example, patients with Crohn's disease who had decreased levels of Faecalibacterium prausnitzii in resected ilea had increased rates of postoperative disease recurrence (17). Using mouse models, the authors demonstrated that a product secreted from $F$. prausnitzii increased colonic levels of IL-10 and provided protection against chemically induced colitis; these results bolstered their claims that this organism has antiinflammatory activities (17). In more recent work, investigators revealed that patients with untreated rheumatoid arthritis had higher fecal levels of Prevotella copri than healthy controls (18). Moreover, mice orally administered $P$. copri had more severe disease in a chemically induced colitis model, a result suggesting that increased abundance of $P$. copri exacerbates inflammatory conditions (18). However, in the case of both F. prausnitzii and P. copri in mouse models, the addition of the organism in question had far-reaching effects on the composition of the microbiota. Thus it remains unclear whether the observed results are directly attribut- 
Lumen

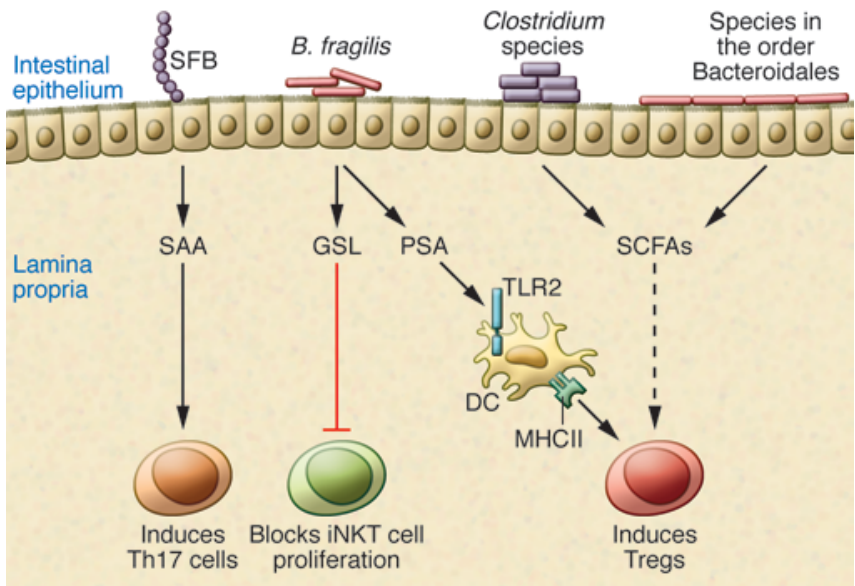

Figure 1. Commensal bacteria that modulate the intestinal immune system. SFB induces Th17 in the intestinal lamina propria via a mechanism that involves host production of serum amyloid $A(S A A)$. $B$. fragilis has pleiotropic effects: it produces a glycosphingolipid (GSL) that inhibits iNKT cell proliferation in the colonic lamina propria; in contrast, $B$. fragilis PSA interacts with TLR2 on DCs to induce colonic Tregs. A group of Clostridium species taken en masse and individual species within the order Bacteroidales (Bacteroides caccae, Bacteroides massiliensis, Bacteroides thetaiotaomicron, Bacteroides vulgatus, and Parabacteroides distasonis) have been demonstrated to induce colonic Tregs, presumably via bacterial production of SCFAs.

able to the administered organism or a consequence of the other changes in the microbiota, with $F$. prausnitzii and $P$. copri serving simply as relevant biomarkers.

In contrast to the capacity of dysbiosis to worsen disease, manipulation of the microbiota in patients with disease can also ameliorate an underlying disorder. In fact, Metchnikoff was among the first who advocated modifying the intestinal flora to improve human health (19). Resurgent interest in the use of fecal transplants for various disease states (e.g., Clostridium difficile colitis, inflammatory bowel disease) is based on the idea that the more normal transplanted flora will replace the diseasepromoting microbiota (20-22). Furthermore, antibiotics have often been used to produce antiinflammatory effects (e.g., azithromycin in patients with cystic fibrosis, sulfasalazine in patients with rheumatoid arthritis and inflammatory bowel disease, dapsone in patients with neutrophilic dermatoses). It is possible that the antiinflammatory effects of these antibiotics are mediated via alterations in the microbiota. Although no study of disease association, candidate organisms, or manipulation has yielded definitive data, these clinical studies en masse strongly suggest that the microbiota is capable of augmenting the immune response and impacting human health.

\section{Identification of commensal bacteria with defined immunomodulatory properties}

It has been known since at least the 1930s that germ-free (GF) mice, which are completely devoid of a microbiota, have a vastly undeveloped immune system compared with that of a WT mouse with a "normal" microbiota $(23,24)$. Not only do GF mice have fewer lymphocytes overall, but their effector $\mathrm{T}$ cells are skewed toward a Th2 phenotype. Remarkably, within 2-3 weeks after a GF animal is given back its normal flora, these defects are largely corrected (24). The microbiota is needed not only for the ontogeny of the immune system but also for its maintenance: antibiotic-treated animals have an immature immune system similar to that of GF animals, with decreased numbers of lymphocytes and diminished cytokine expression $(24,25)$. Our laboratory has demonstrated exquisite host specificity between the source of the microbiota and its ability to induce maturation of the small-intestinal immune system. In contrast to gnotobiotic mice that were colonized with a normal mouse microbiota and had a small-intestinal immune system comparable to that of specific pathogen-free (SPF) mice, gnotobiotic mice colonized with a normal microbiota from either humans or rats had an immature small-intestinal immune system that was indistinguishable from that of GF mice (8). Taken together, these data indicate that animals and their microbiota have coevolved and that a constant dialogue between the two is needed for maintenance of the immune system.

What has become clear over the past decade is that not all bacteria within the microbiota affect the immune system; rather, specific bacteria have particular immunomodulatory effects (Figure 1). Recent efforts have focused on identifying these bacteria with immunomodulatory properties, and this area has been extensively reviewed (26-30). Our laboratory identified the first such commensal organism, demonstrating that Bacteroides fragilis - via production of a single polysaccharide referred to as polysaccharide $\mathrm{A}$ (PSA) - is able to restore Th1/Th2 balance in GF mice (31). Further work demonstrated that PSA is both protective and therapeutic in murine models of colitis and multiple sclerosis via induction of IL-10-secreting Tregs in a process that requires both TLR2 and MHCII (32-36). More recently, we and others have independently established that $B$. fragilis also produces glycosphingolipids that affect invariant natural killer T (iNKT) cells $(37,38)$. Although other investigators showed that these glycosphingolipids activate iNKT cells in vitro (38), we found that they inhibit endogenous iNKT cell agonists both in vitro and in vivo (37). These discrepant results may be related to differences in the tested molecules resulting from variations in the purification schemes. Moreover, we demonstrated that $B$. fragilis-produced glycosphingolipids decrease the number of iNKT cells in the colonic lamina propria and that this effect leads to improved outcomes in a murine colitis model (37). Figure 2 provides an overview of the multiple immunomodulatory effects conferred by $B$. fragilis.

Other researchers have found that a group of 46 murine Clostridium species as well as a group of 17 human Clostridium species can induce Tregs in the colonic lamina propria of mice, with consequent protection in murine models of colitis and allergy (39, 40). Interestingly, when individually administered to GF mice, these Clostridium species had little or no effect on Tregs, an observation that indicates that the larger community of organisms cooperates in induction of Tregs. While these findings initially suggested that Clostridium species were critical for the induction of Tregs, it appears that this process may be a function common to many commensal bacteria. A recent report demonstrated that monocolonization of mice with any of 5 bacterial species - all from the order Bacteroidales - results in potent induction of 


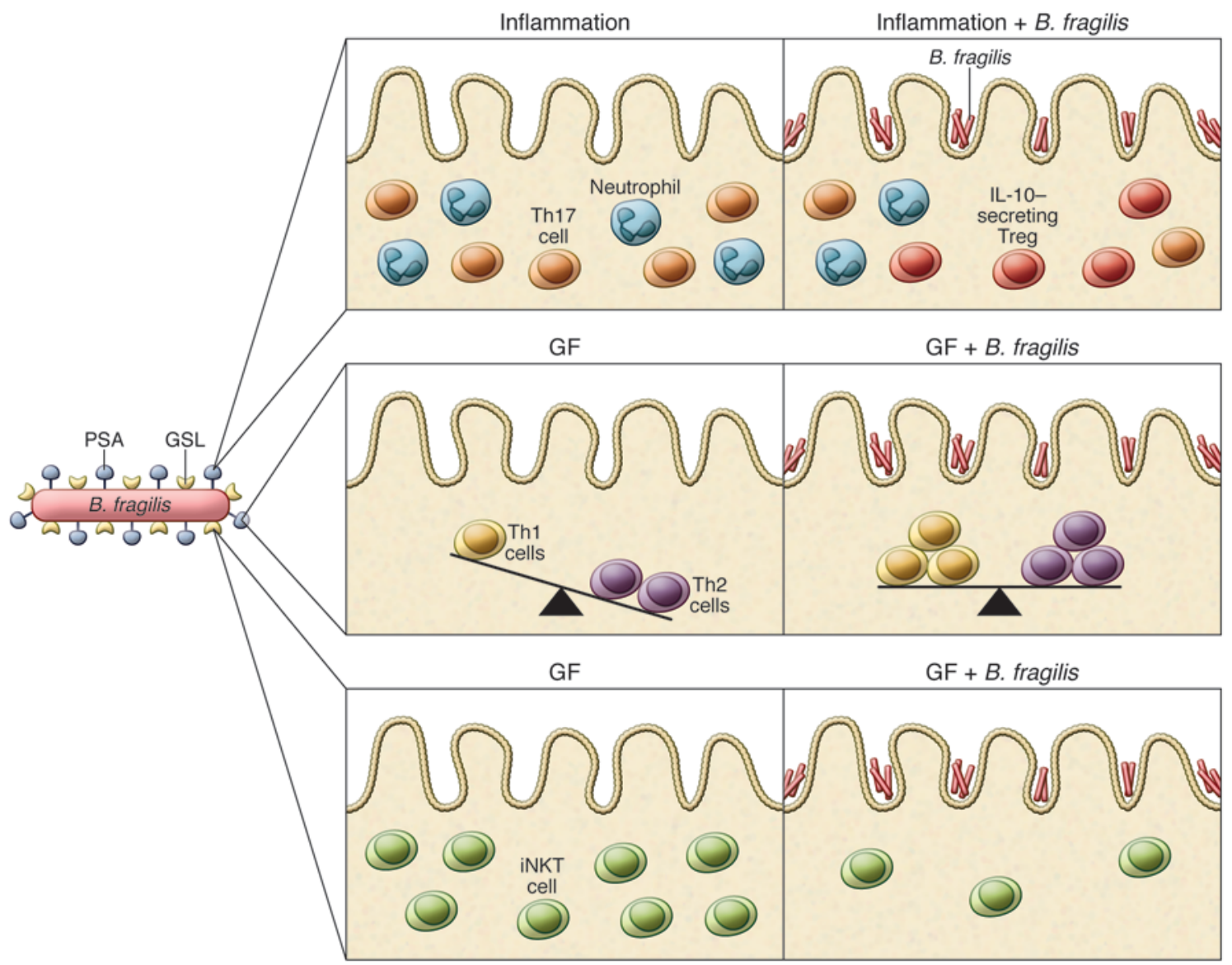

Figure 2. B. fragilis has multiple immunomodulatory effects. $B$. fragilis-produced PSA induces colonic IL-10-secreting Tregs only after inflammatory insults $(34,50)$ (top panel) and restores the balance of systemic Th1/Th2 cells in GF mice (31) (middle panel), thereby playing a protective role in murine models of colitis and multiple sclerosis (32-34, 51). B. fragilis also expresses GSLs, such as $\alpha$-GalCer, that inhibit iNKT cell proliferation (37) (bottom panel).

colonic Tregs, though the biological significance of this increase remains to be demonstrated (41). Furthermore, 2 research teams independently identified a Clostridium-related organism that still has not been cultured - designated segmented filamentous bacteria (SFB) - as critical for induction of Th17 cells in the smallintestinal lamina propria; 1 group also demonstrated that SFB colonization increases small-intestinal and colonic Tregs $(42,43)$. In addition to altering the numbers of specific cell types, immunomodulatory bacteria can influence the activation threshold of the immune system (44).

Given the presence of more than 1,000 bacterial species in the human intestine, these limited examples merely serve as proof of concept that certain commensal bacteria have specific immunomodulatory properties. There are undoubtedly numerous other undiscovered examples that affect the entire panoply of immune cells - not just Tregs, Th17 cells, and iNKT cells. Moreover, we are still at the very early stages of defining the normal viral and fungal constituents of the microbiome. Almost certainly, these components of the microbiota also affect development of the immune system in ways we do not yet understand. Even though at present there are few specifically identified examples of immunomodulatory commensal bacteria, several patterns have emerged that allow a glimpse into how these organisms influence the immune system.

\section{Overview of microbe-induced maturation of the intestinal immune system}

As depicted in Figure 3, many steps are likely to be involved in bacteria-mediated development of the immune system. Although the specific details have remained largely elusive, the host must recognize the bacteria or bacterial product(s), T cells must proliferate within the intestinal system, and the TCR repertoire must undergo maturation. Much work has focused on the role of pattern recognition receptors (PRRs) in the detection of bacteria. Intestinal expression of PRRs is known to be involved in intestinal homeostasis and control of immune-mediated diseases $(45,46)$. However, analysis of the intestinal immune system in mice deficient in MyD88, an adaptor protein for virtually all the TLR pathways, or Nod2, an important intracellular sensor for bacteria, detected decreases only in numbers of TCR $\gamma \delta$ and CD $8 \alpha \alpha \operatorname{TCR} \alpha \beta$ intraepithelial lymphocytes $(47,48)$; these results suggested that the MyD88- and Nod2-dependent pathways play only a minor role in homeostasis of the mucosal immune system.

Although MyD88-deficient mice had defects only in the intraepithelial lymphocyte compartment, the increase in Tregs mediated by B. fragilis PSA requires signaling through TLR2 - a MyD88-dependent pathway - expressed on DCs (36); the discrepancy between these results may relate to the fact that $B$. fragilis typically is not present in the murine microbiota and that its modulation of mucosal 


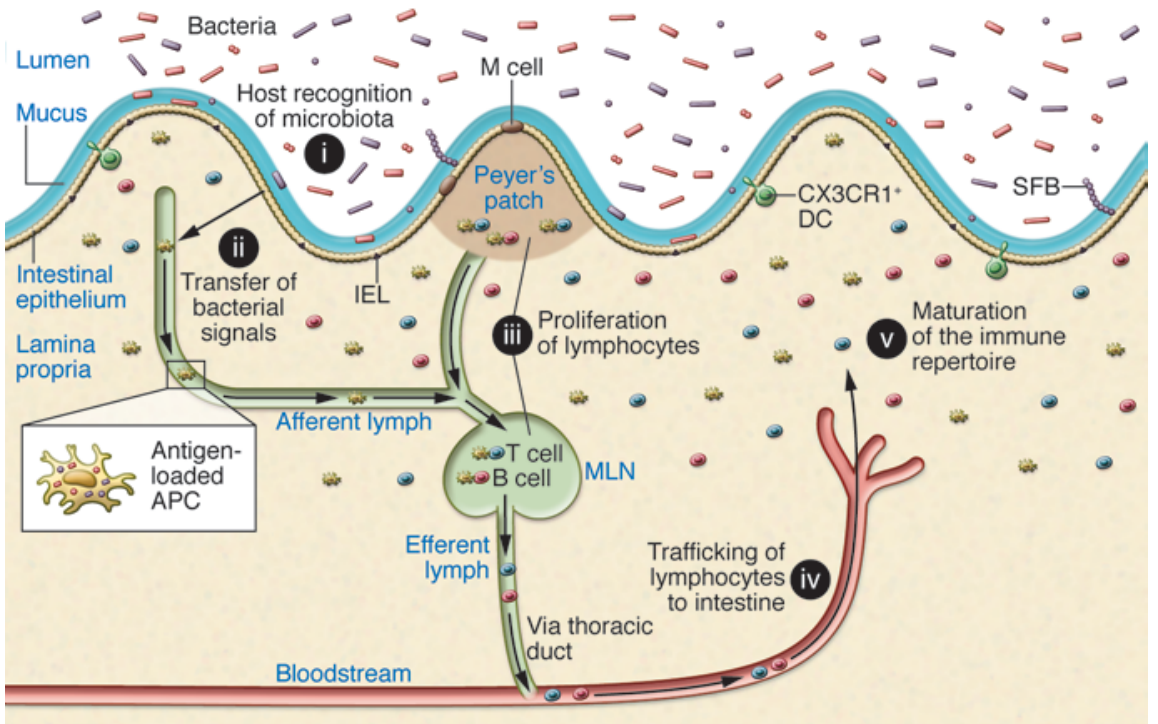

Figure 3. A mechanistic model of microbeinduced maturation of the intestinal immune system. A portion of the small intestine is depicted. The majority of the microbiota are luminal, some organisms reside in the mucus layer, and only a few bacteria adhere to the epithelial surface. Immunogenic signals from the gut flora are recognized by the host (i) and transmitted across the epithelium (ii). In a process likely involving APCs, these signals reach the secondary lymphoid organs, where APC-lymphocyte interactions result in lymphocyte proliferation (iii). Lymphocytes leave the mesenteric lymph nodes (MLN) through the efferent lymphatic vessels, go through the systemic circulation, and migrate back to the intestine (iv). The resulting intestinal lymphocytes disperse throughout the lamina propria and intraepithelial layer and reflect the mature immune repertoire of the intestinal immune system (v). IEL, intraepithelial lymphocyte. immunity is most prominent during inflammation $(49,50)$. Although in vitro experiments suggested that PSA can induce IL-10 production by directly stimulating TLR2-expressing T cells in an APC-independent manner (35), the in vivo relevance of this observation is unclear, since it is not readily apparent how $\mathrm{T}$ cells in the lamina propria interact with a luminal antigen without help from APCs. Recently, it has been demonstrated that dendritic cells - specifically plasmacytoid dendritic cells - are absolutely required in vivo for PSA-mediated IL-10 production $(51,52)$, clarifying that APCs are, in fact, critical for PSA-mediated immunoregulation. In contrast to the TLR2-dependence of PSA, studies on SFB induction of Th17 cells revealed that this process is independent of MyD88, and the PRR adaptors toll-like receptor adaptor molecule 2 (TRIF) and receptor (TNFRSF)-interacting serine-threonine kinase 2 (RIP2). Similarly, clostridial stimulation of Tregs is independent of MyD88, RIP2, and caspase recruitment domain family member 9 (CARD9) (40). These data indicate that PRRs may contribute to recognition of some immunomodulatory bacteria but that other, yet-unidentified pathways exist as well.

After the host recognizes the bacteria, the transfer of immunogenic signals to the lamina propria, which eventually leads to $\mathrm{T}$ cell proliferation, must take place. This dissemination of the signal likely involves APCs, as antigen-loaded APCs are known to prime and activate naive lymphocytes. Given that intraepithelial and lamina propria lymphocytes originate from mesenteric lymph nodes and Peyer's patches $(53,54)$, the relevant APC-T cell interactions leading to $\mathrm{T}$ cell proliferation probably occur in these secondary lymphoid organs. Ultimately, T cell proliferation is thought to lead to the transition from the polyclonal TCR repertoire found in infancy to the oligoclonal repertoire found in adulthood (55). It remains to be determined whether this oligoclonal repertoire is fixed over time and how it is affected by alterations in the microbiota (e.g., as a result of enteric infections or antibiotic treatment).

\section{Mechanistic principles underlying commensal- mediated immunomodulation}

Although the specifics underlying host-commensal interactions remain largely enigmatic, some aspects of the downstream mechanistic details are beginning to be clarified. The homeostasis of
iNKT cells represents one example of bacterial immunomodulation involving multiple mechanisms. Although $B$. fragilis modulates colonic iNKT cell levels by affecting their local proliferation (37), the greater number of iNKT cells in the lungs and colonic lamina propria of GF mice than in those of SPF mice is due to aberrant levels of CXCL16, a chemokine important for iNKT cell migration and homeostasis (56). Commensal bacteria regulate pulmonary and colonic levels of CXCL16 epigenetically by decreasing the methylation status of the Cxcl16 gene (56).

Epigenetic changes - specifically, acetylation of the Foxp3 gene - have also been implicated in the homeostasis of colonic Tregs. In this case, short-chain fatty acids (SCFAs), which are common bacterial metabolites, inhibit histone deacetylase and lead to an increase in Foxp3 expression (57-59). Although it is clear that SCFAs induce colonic Tregs, the specific details are a bit murky because of significant differences among studies (possibly due to technical differences). One group demonstrated that propionate and acetate exerted effects stronger than those of butyrate on the induction of thymically derived (i.e., Helios ${ }^{+}$) Tregs in the colon via enhanced proliferation within and trafficking to the colon (59). Two other groups demonstrated that butyrate had a greater effect than propionate (acetate being inactive) in promoting differentiation of extrathymically derived (i.e., Helios- or neuropilin-1-1) Tregs, with no discernible effect on thymically derived Tregs $(57,58)$. All 3 studies agree, however, that SCFAs act by inhibition of histone deacetylase, which leads to increased acetylation and expression of Foxp3. Ultimately, it may be that these different SCFAs act slightly differently from one another, with butyrate influencing de novo generation of colonic Tregs, acetate inducing accumulation of thymically derived Tregs in the colon, and propionate performing both functions (57). Along these lines, mice colonized with bacteria known to induce colonic Tregs have elevated cecal levels of these SCFAs. Three Bacteroides species (B. caccae, B. massiliensis, and B. thetaiotaomicron) increased levels of acetate and propionate, whereas Parabacteroides distasonis and the mix of 17 humanderived Clostridium species elevated levels of all 3 SCFAs (39, 41). Notably, it has not been demonstrated for any of these 
organisms that microbe-induced SCFA production is critical for Treg induction or whether these bacteria have other redundant mechanisms for modulating the immune system.

\section{Location, location, location}

Given that the first step of microbe-induced maturation of the intestinal immune system likely involves host recognition of the bacteria, it is significant - but still unclear - whether the location of the bacteria (e.g., mucosa-associated, luminal) is important to this process. Intuitively, one might assume that immunomodulatory bacteria would need to be close to the epithelial surface to interact with the host. Indeed, B. fragilis, SFB, and the immunoregulatory Clostridium species are all tightly associated with the intestinal epithelium $(35,39,40,60)$. It is unclear whether the consistency within this small sample size should be taken to suggest that mucosally associated bacteria are more relevant than luminal bacteria for immunomodulation. Although some have suggested that biopsy samples are preferable to fecal samples for identification of immunoregulatory bacteria $(61,62)$, all current examples of such bacteria identified in screens were isolated from fecal samples (39, $40,42,43)$. This fact is perhaps not terribly surprising given that the fecal bacterial community represents a combination of luminal bacteria and shed, mucosally adherent bacteria (63). Although it has been demonstrated that the intestinal microbiota can affect immune responses at distant sites (e.g., in the CNS, joints, and lungs) $(34,56,64-66)$, recent work has revealed that local microbial colonization is critical for induction of both effector and regulatory T cells in the skin (67). Using a combination of gnotobiotic animals and antibiotic treatment, the investigators elegantly demonstrated that colonization of the skin - but not the gut with Staphylococcus epidermidis was sufficient to normalize cutaneous levels of Th1 and Th17 cells (67). In short, while we know that the gastrointestinal microbiota has the capacity to modulate many facets of systemic immunity, we are still learning about how local microbial niches are involved.

\section{Timing matters}

The hygiene hypothesis, which is supported by epidemiologic associations, contends that microbial exposures early in life influence immune responses later in life $(11,68,69)$. In fact, some clinical studies have suggested that even prenatal exposures shape development of the immune system $(70,71)$, potentially because of microbes present in the placenta (72). Although the vast majority of the immunologic defects in a GF mouse are corrected once the mouse is "conventionalized" by receipt of its normal microbiota, there is a time-sensitive window early in life when hostmicrobial interactions are required for the homeostatic control of certain immune cell populations. Once this window has closed, subsequent manipulation of the microbiota fails to redress the initial deficiency, culminating in examples of "original sin." For example, GF mice conventionalized as adults (GF/a mice) have high numbers of iNKT cells in the colonic lamina propria and lung similar to those in GF mice; in contrast, GF mice conventionalized on day 1 of life (GF/n mice) have low levels of colonic and pulmonary iNKT cells similar to those in SPF mice (56). Moreover, GF/n mice have better outcomes in models of colitis and asthma than do GF/a mice (56), a result lending direct experimental support to the hygiene hypothesis. Recent evidence has demonstrated that like numbers of iNKT cells - numbers of colonic CD4 $4^{+}$lymphoid tissue inducer-like cells, which are lower in GF mice than in SPF mice, are not normalized in GF/a mice (73). Collectively, these findings suggest the intriguing possibility that there exists a "teleologic imperative" for certain innate immune cells that represent the first line of defense against infection and inflammation - i.e., that they are "instructed" early in life and are not subject to the plasticity of the microbiota. In contrast, the adaptive immune system, which must be able to respond to a changing environment, changes in concert with the microbial population.

\section{Context is everything}

Although commensal microbes typically live in symbiotic harmony with the host, these organisms (e.g., Staphylococcus species, Streptococcus species) - when they escape their normal habitats and interact with the host in a new context - are among the most common causes of infectious diseases. For example, B. fragilis is a commensal readily found in the human colon, but it is also the most commonly isolated organism from cases of intra-abdominal abscesses $(74,75)$. These rather disparate effects are both mediated by PSA and depend on the context of its interaction with the host $(30,35,76)$. Moreover, although PSA induces Tregs, it does so only under settings of inflammation with virtually no effect on Tregs in the healthy state $(34,50)$. This finding is in stark contrast to Clostridium species, which induce colonic Tregs in both the healthy and the inflamed state $(39,40)$. In thinking about potential pharmaceutical implications, it may be preferable to have agents - such as PSA - that exert their effect only when needed as opposed to altering the homeostatic immune system and potentially causing untoward effects. In addition to considering whether the host is inflamed, the cause and/or type of inflammation may also be important in modulating the microbe-induced phenotype. For example, SFB induces Th17 cells, which are typically considered proinflammatory cells. As such, colonization with SFB is associated with worse outcomes in murine models of rheumatoid arthritis and multiple sclerosis $(64,66)$. In contrast, using a murine model of type 1 diabetes, which reflects an inflammatory process different from that of the other models of autoimmunity (77), the presence of SFB is associated with disease protection (78). Taken together, these studies highlight that the underlying context of host-commensal interactions can have a profound impact on how microbe-induced immunoregulation manifests in the host.

\section{Parting thoughts}

Over the past 25 years, the hygiene hypothesis has evolved to suggest that microbial exposures can modulate disease incidence and/or severity in genetically predisposed patients (79-81). In many of these cases, the differences in phenotype clearly result from commensal-induced immunomodulation; however, our understanding of how commensal microbes influence the ontogeny and maintenance of the immune system is still in its infancy: examples of commensal bacteria with defined immunomodulatory properties are still limited, only 2 relevant bacterial molecules - both from $B$. fragilis - have been identified thus far, and we know virtually nothing about how the nonbacterial components of the microbiota affect the immune system. Essentially, 
our progress in this field has brought us to a point similar to that at which studies of pathogens had advanced a century ago, with limited, somewhat serendipitous successes. While current work is focused on the use of reductionist approaches to gain a better understanding of host-commensal interactions, we ultimately need to study these connections in a more natural community context, in line with that now evident in studies of microbial pathogenesis (82-84). Many fundamental questions remain unresolved, including how the host integrates multiple counteracting immunomodulatory signals, how immunoregulatory signals provided within the gut are transmitted to extraintestinal sites, and how the microbiota can be manipulated in a meaningful manner to positively affect human health. Despite these limitations, the early successes in this field have provided conceptual proof of the importance of the microbiota in regulation of the immune system and have opened the door for potentially unlimited therapeutic applications arising from a better under- standing of these host-commensal interactions. We are poised to make major strides in the coming years. Perhaps the results will be described in a sequel to Microbe Hunters that will detail the extraordinary efforts of the pioneers in these early days of research on the microbiota.

\section{Acknowledgments}

We thank Julie B. McCoy for editorial assistance. N.K. Surana is supported by NIH grant K08 AI108690 and a Faculty Career Development Award from Boston Children's Hospital. Work in D.L. Kasper's laboratory is supported in part by a senior research award from the Crohn's \& Colitis Foundation of America.

Address correspondence to: Dennis L. Kasper, Department of Microbiology and Immunobiology, Harvard Medical School, 77 Avenue Louis Pasteur, Room 1056, Boston, Massachusetts 02115, USA. Phone: 617.432.5505; E-mail: dennis_kasper@hms.harvard.edu.
1. De Kruif P. Microbe Hunters. New York, New York, USA: Harcourt, Inc.; 1926.

2. Kendall AI. Some observations on the study of the intestinal bacteria. J Biol Chem. 1909;6:499-507.

3. Hooper LV, Gordon JI. Commensal hostbacterial relationships in the gut. Science. 2001;292(5519):1115-1118.

4. Hooper LV, Wong MH, Thelin A, Hansson L, Falk PG, Gordon JI. Molecular analysis of commensal host-microbial relationships in the intestine. Science. 2001;291(5505):881-884.

5 . Luckey TD. Introduction to intestinal microecology. Am J Clin Nutr. 1972;25(12):1292-1294.

6. Smith MI, et al. Gut microbiomes of Malawian twin pairs discordant for kwashiorkor. Science. 2013;339(6119):548-554.

7. van der Waaij D, Berghuis-de Vries JM. Colonization resistance of the digestive tract in conventional and antibiotic-treated mice. J Hyg (Lond). 1971;69(3):405-411.

8. Chung H, et al. Gut immune maturation depends on colonization with a host-specific microbiota. Cell. 2012;149(7):1578-1593.

9. Bhatt AS, et al. Sequence-based discovery of Bradyrhizobium enterica in cord colitis syndrome. N Engl JMed. 2013;369(6):517-528.

10. de Goffau MC, et al. Fecal microbiota composition differs between children with beta-cell autoimmunity and those without. Diabetes. 2013;62(4):1238-1244.

11. Ege MJ, et al. Exposure to environmental microorganisms and childhood asthma. N Engl JMed. 2011;364(8):701-709.

12. Huang YJ, et al. The airway microbiome in severe asthma. Ann Am Thorac Soc. 2014;11(suppl 1):S78.

13. Morgan XC, et al. Dysfunction of the intestinal microbiome in inflammatory bowel disease and treatment. Genome Biol. 2012;13(9):R79.

14. Wang Y, Kasper LH. The role of microbiome in central nervous system disorders. Brain Behav Immun. 2014;38:1-12.

15. Wong BC, et al. Helicobacter pylori eradication to prevent gastric cancer in a high-risk region of China: a randomized controlled trial. JAMA. 2004;291(2):187-194.

16. Xuan C, et al. Microbial dysbiosis is associ- ated with human breast cancer. PLoS One. 2014;9(1):e83744.

17. Sokol H, et al. Faecalibacterium prausnitzii is an anti-inflammatory commensal bacterium identified by gut microbiota analysis of Crohn disease patients. Proc Natl Acad Sci U S A. 2008;105(43):16731-16736.

18. Scher JU, et al. Expansion of intestinal Prevotella copri correlates with enhanced susceptibility to arthritis. Elife. 2013;2:e01202.

19. Metchnikoff E. The Prolongation OfLife: Optimistic Studies. New York, New York, USA: G.P. Putnam's Sons; 1908.

20. Pamer EG. Fecal microbiota transplantation: effectiveness, complexities, and lingering concerns. Mucosal Immunol. 2014;7(2):210-214.

21. van Nood E, et al. Duodenal infusion of donor feces for recurrent Clostridium difficile. $\mathrm{NEnglJ}$ Med. 2013;368(5):407-415.

22. Zhang FM, Wang HG, Wang M, Cui BT, Fan ZN, Ji GZ. Fecal microbiota transplantation for severe enterocolonic fistulizing Crohn's disease. World J Gastroenterol. 2013;19(41):7213-7216.

23. Glimstedt G. Bakterienfreie Meerscheinchen. Acta Pathol Microbiol Scand. 1936;30(1):1-295.

24. Gordon HA. Morphological and physiological characterization of germfree life. Ann N Y Acad Sci. 1959;78:208-220.

25. Hill DA, et al. Metagenomic analyses reveal antibiotic-induced temporal and spatial changes in intestinal microbiota with associated alterations in immune cell homeostasis. Mucosal Immunol. 2010;3(2):148-158.

26. Honda K, Littman DR. The microbiome in infectious disease and inflammation. Annu Rev Immunol. 2012;30:759-795.

27. Hooper LV, Littman DR, Macpherson AJ. Interactions between the microbiota and the immune system. Science. 2012;336(6086):1268-1273.

28. Ivanov II, Honda K. Intestinal commensal microbes as immune modulators. Cell Host Microbe. 2012;12(4):496-508.

29. Kamada N, Nunez G. Role of the gut microbiota in the development and function of lymphoid cells. J Immunol. 2013;190(4):1389-1395.

30. Surana NK, Kasper DL. The yin yang of bacterial polysaccharides: lessons learned from $B$. fragilis PSA. Immunol Rev. 2012;245(1):13-26.

31. Mazmanian SK, Liu CH, Tzianabos AO, Kasper DL. An immunomodulatory molecule of symbiotic bacteria directs maturation of the host immune system. Cell. 2005;122(1):107-118.

32. Mazmanian SK, Round JL, Kasper DL. A microbial symbiosis factor prevents intestinal inflammatory disease. Nature. 2008;453(7195):620-625.

33. Ochoa-Reparaz J, et al. Central nervous system demyelinating disease protection by the human commensal Bacteroides fragilis depends on polysaccharide A expression. Jimmunol. 2010;185(7):4101-4108.

34. Ochoa-Reparaz J, et al. A polysaccharide from the human commensal Bacteroides fragilis protects against CNS demyelinating disease. Mucosal Immunol. 2010;3(5):487-495.

35. Round JL, et al. The Toll-like receptor 2 pathway establishes colonization by a commensal of the human microbiota. Science. 2011;332(6032):974-977.

36. Wang Q, et al. A bacterial carbohydrate links innate and adaptive responses through Toll-like receptor 2. JExp Med. 2006;203(13):2853-2863.

37. An D, et al. Sphingolipids from a symbiotic microbe regulate homeostasis of host intestinal natural killer T cells. Cell. 2014;156(1-2):123-133.

38. Wieland Brown LC, et al. Production of $\alpha$-galactosylceramide by a prominent member of the human gut microbiota. PLoS Biol. 2013;11(7):e1001610.

39. Atarashi $\mathrm{K}$, et al. Treg induction by a rationally selected mixture of Clostridia strains from the human microbiota. Nature. 2013;500(7461):232-236.

40. Atarashi K, et al. Induction of colonic regulatory $\mathrm{T}$ cells by indigenous Clostridium species. $\mathrm{Sci}$ ence. 2011;331(6015):337-341.

41. Faith JJ, Ahern PP, Ridaura VK, Cheng J, Gordon JI. Identifying gut microbe-host phenotype relationships using combinatorial communities in gnotobiotic mice. Sci Transl Med. 2014;6(220):220ra211.

42. Gaboriau-Routhiau V, et al. The key role of segmented filamentous bacteria in the coordinated 
maturation of gut helper $\mathrm{T}$ cell responses. Immunity. 2009;31(4):677-689.

43. Ivanov II, et al. Induction of intestinal Th17 cells by segmented filamentous bacteria. Cell. 2009;139(3):485-498.

44. Chappert P, Bouladoux N, Naik S, Schwartz RH. Specific gut commensal flora locally alters $\mathrm{T}$ cell tuning to endogenous ligands. Immunity. 2013;38(6):1198-1210.

45. Rakoff-Nahoum S, Paglino J, Eslami-Varzaneh F, Edberg S, Medzhitov R. Recognition of commensal microflora by toll-like receptors is required for intestinal homeostasis. Cell. 2004;118(2):229-241.

46. Wen $\mathrm{L}$, et al. Innate immunity and intestinal microbiota in the development of Type 1 diabetes. Nature. 2008;455(7216):1109-1113.

47. Jiang W, et al. Recognition of gut microbiota by NOD2 is essential for the homeostasis of intestinal intraepithelial lymphocytes. JExp Med. 2013;210(11):2465-2476.

48. Yu Q, Tang C, Xun S, Yajima T, Takeda K, Yoshikai Y. MyD88-dependent signaling for IL-15 production plays an important role in maintenance of CD8 alpha alpha TCR alpha beta and TCR gamma delta intestinal intraepithelial lymphocytes. J Immunol. 2006;176(10):6180-6185.

49. Lee SM, Donaldson GP, Mikulski Z, Boyajian S, Ley K, Mazmanian SK. Bacterial colonization factors control specificity and stability of the gut microbiota. Nature. 2013;501(7467):426-429.

50. Round JL, Mazmanian SK. Inducible Foxp3+ regulatory T-cell development by a commensal bacterium of the intestinal microbiota. Proc Natl Acad Sci U S A. 2010;107(27):12204-12209.

51. Dasgupta S, Erturk-Hasdemir D, Ochoa-Reparaz J, Reinecker HC, Kasper DL. Plasmacytoid dendritic cells mediate anti-inflammatory responses to a gut commensal molecule via both innate and adaptive mechanisms. Cell Host Microbe. 2014;15(4):413-423.

52. Shen Y, Giardino Torchia ML, Lawson GW, Karp CL, Ashwell JD, Mazmanian SK. Outer membrane vesicles of a human commensal mediate immune regulation and disease protection. Cell Host Microbe. 2012;12(4):509-520.

53. Guy-Grand D, Griscelli C, Vassalli P. The gut-associated lymphoid system: nature and properties of the large dividing cells. Eur J Immunol. 1974;4(6):435-443.

54. Guy-Grand D, Griscelli C, Vassalli P. The mouse gut $\mathrm{T}$ lymphocyte, a novel type of $\mathrm{T}$ cell. Nature, origin, and traffic in mice in normal and graft-versus-host conditions. J Exp Med. 1978;148(6):1661-1677.
55. Williams AM, et al. Intestinal alpha beta $\mathrm{T}$ cells differentiate and rearrange antigen receptor genes in situ in the human infant. JImmunol. 2004;173(12):7190-7199.

56. Olszak T, et al. Microbial exposure during early life has persistent effects on natural killer $\mathrm{T}$ cell function. Science. 2012;336(6080):489-493.

57. Arpaia N, et al. Metabolites produced by commensal bacteria promote peripheral regulatory T-cell generation. Nature. 2013;504(7480):451-455.

58. Furusawa $Y$, et al. Commensal microbe-derived butyrate induces the differentiation of colonic regulatory T cells. Nature. 2013;504(7480):446-450.

59. Smith PM, et al. The microbial metabolites, short-chain fatty acids, regulate colonic Treg cell homeostasis. Science. 2013;341(6145):569-573.

60. Davis CP, Savage DC. Habitat, succession, attachment, and morphology of segmented, filamentous microbes indigenous to the murine gastrointestinal tract. Infect Immun. 1974;10(4):948-956.

61. Fraher MH, O'Toole PW, Quigley EM. Techniques used to characterize the gut microbiota: a guide for the clinician. Nat Rev Gastroenterol Hepatol. 2012;9(6):312-322.

62. Vujkovic-Cvijin I, et al. Dysbiosis of the gut microbiota is associated with hiv disease progression and tryptophan catabolism. Sci Transl Med. 2013;5(193):193ra191.

63. Eckburg PB, et al. Diversity of the human intestinal microbial flora. Science. 2005;308(5728):1635-1638.

64. Lee YK, Menezes JS, Umesaki Y, Mazmanian SK. Proinflammatory T-cell responses to gut microbiota promote experimental autoimmune encephalomyelitis. Proc Natl Acad Sci U S A. 2011;108(suppl 1):4615-4622.

65. Noverr MC, Falkowski NR, McDonald RA, McKenzie AN, Huffnagle GB. Development of allergic airway disease in mice following antibiotic therapy and fungal microbiota increase: role of host genetics, antigen, and interleukin- 13 . Infect Immun. 2005;73(1):30-38.

66. Wu HJ, et al. Gut-residing segmented filamentous bacteria drive autoimmune arthritis via $\mathrm{T}$ helper 17 cells. Immunity. 2010;32(6):815-827.

67. Naik S, et al. Compartmentalized control of skin immunity by resident commensals. Science. 2012;337(6098):1115-1119.

68. Bach JF. The effect of infections on susceptibility to autoimmune and allergic diseases. $N$ EnglJ Med. 2002;347(12):911-920.

69. Strachan DP. Hay fever, hygiene, and household size. BMJ. 1989;299(6710):1259-1260.

70. Ege MJ, et al. Prenatal farm exposure is related to the expression of receptors of the innate immunity and to atopic sensitization in school-age children. JAllergy Clin Immunol. 2006;117(4):817-823.

71. Ege MJ, et al. Specific IgE to allergens in cord blood is associated with maternal immunity to Toxoplasma gondii and rubella virus. Allergy. 2008;63(11):1505-1511.

72. Aagaaard K, Ma J, Antony KM, Ganu R, Petrosino J, Versalovic J. The placenta harbors a unique microbiome. Sci Transl Med. 2014;6(237):237ra265.

73. Chen VL, Surana NK, Duan J, Kasper DL. Role of murine intestinal interleukin-1 receptor 1-expressing lymphoid tissue inducer-like cells in salmonella infection. PLoS One. 2013;8(6):e65405.

74. Gorbach SL, Bartlett JG. Anaerobic infections (second of three parts). N EnglJ Med. 1974;290(22):1237-1245.

75. Polk BF, Kasper DL. Bacteroides fragilis subspecies in clinical isolates. Ann Intern Med. 1977;86(5):569-571.

76. Tzianabos AO, Onderdonk AB, Rosner B, Cisneros RL, Kasper DL. Structural features of polysaccharides that induce intra-abdominal abscesses. Science. 1993;262(5132):416-419.

77. Mathis D, Benoist C. Microbiota and autoimmune disease: the hosted self. Cell Host Microbe. 2011;10(4):297-301.

78. Kriegel MA, Sefik E, Hill JA, Wu HJ, Benoist C, Mathis D. Naturally transmitted segmented filamentous bacteria segregate with diabetes protection in nonobese diabetic mice. Proc Natl Acad Sci U S A. 2011;108(28):11548-11553.

79. Cadwell K, et al. Virus-plus-susceptibility gene interaction determines Crohn's disease gene Atg16L1 phenotypes in intestine. Cell. 2010;141(7):1135-1145.

80. Caliskan M, et al. Rhinovirus wheezing illness and genetic risk of childhood-onset asthma. N Engl JMed.2013;368(15):1398-1407.

81. Yatsunenko $\mathrm{T}$, et al. Human gut microbiome viewed across age and geography. Nature. 2012;486(7402):222-227.

82. Basler M, Ho BT, Mekalanos JJ. Tit-for-tat: type VI secretion system counterattack during bacterial cell-cell interactions. Cell. 2013;152(4):884-894.

83. Lysenko ES, Ratner AJ, Nelson AL, Weiser JN. The role of innate immune responses in the outcome of interspecies competition for colonization of mucosal surfaces. PLoS Pathog. 2005;1(1):e1.

84. Winter SE, et al. Gut inflammation provides a respiratory electron acceptor for Salmonella. Nature. 2010;467(7314):426-429. 